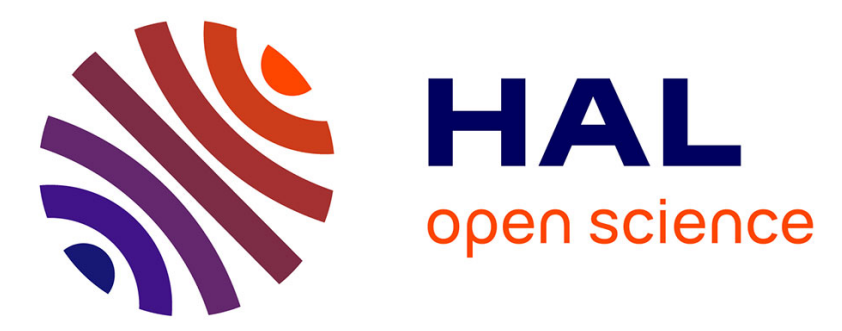

\title{
Mixed FEM for solving a plate type model intended for analysis of pavements with discontinuities
}

\author{
Hanan Nasser, Olivier Chupin, Jean Michel Piau, Armelle Chabot
}

\section{To cite this version:}

Hanan Nasser, Olivier Chupin, Jean Michel Piau, Armelle Chabot. Mixed FEM for solving a plate type model intended for analysis of pavements with discontinuities. Road Materials and Pavement Design, 2018, 3 (19), pp.496-510. 10.1080/14680629.2018.1418653 . hal-01713899

\section{HAL Id: hal-01713899 \\ https://hal.science/hal-01713899}

Submitted on 18 Jun 2020

HAL is a multi-disciplinary open access archive for the deposit and dissemination of scientific research documents, whether they are published or not. The documents may come from teaching and research institutions in France or abroad, or from public or private research centers.
L'archive ouverte pluridisciplinaire HAL, est destinée au dépôt et à la diffusion de documents scientifiques de niveau recherche, publiés ou non, émanant des établissements d'enseignement et de recherche français ou étrangers, des laboratoires publics ou privés. 
Mixed FEM for solving a plate type model intended for analysis of pavements with discontinuities

H. Nasser, O. Chupin, J.-M. Piau, A. Chabot

IFSTTAR, MAST department, Nantes, France

Corresponding author: O. Chupin (email: olivier.chupin@ifsttar.fr)

Provide short biographical notes on all contributors here if the journal requires them. 


\title{
Mixed FEM for solving a plate type model intended for analysis of pavements with discontinuities
}

\begin{abstract}
This paper aims at presenting the development of a numerical tool dedicated to the computation of the mechanical response of pavements incorporating vertical cracks and/or interlayer debonding. In this tool, the structure is modelled as a pilling of "plate" elements of type M4-5n (Multi-Particle Model for Multilayer Materials) which considers 5 equilibrium equations per layer (n stands for the number of layer). Here we focus on the development of a mixed Finite Element (FE) method dedicated to the solving of M4-5n. This method relies on the derivation of a variational principle based on the complementary energy theorem. Expressing stationarity of the functional obtained with respect to all its fields leads to the mixed formulation. Special attention is paid to the discretization process of this formulation in order to avoid ill-conditioned system of algebraic equations after discretization and to insure stability of the solution. The developed method is implemented in a FreeFem++ script. The advantage of the method is twofold: (i) the initial 3D problem can be handled through 2D FE simulations and (ii) finite values of the generalized efforts are obtained at crack and interlayer debonding locations. This approach is thus particularly adapted to parametric studies and, in the future, might be considered for crack growth in layered structures such as pavements. This paper ends with the analysis by means of M4-5n of a 3D structure incorporating cracks, representative of a pavement tested under full-scale conditions during an accelerated fatigue test performed at IFSTTAR. Several scenarios of cracking are analysed and compared to experimental results.
\end{abstract}

Keywords: Pavement; Multilayer Structure; M4-5n; Mixed Finite Elements; Cracking; Debonding

\section{Introduction}

A pavement is a multilayer structure resting on a soil foundation. It can undergo distress due to many phenomena resulting in several types of degradation among which are vertical cracking across layers or partial/total interlayer debonding (Chabot et al., 2016). To study these mechanisms, existing advanced models are generally based on threedimensional continuum mechanics and related to the fracture or the damage theory (Pommier et al., 2009). The elastic frame is generally retained to model the constitutive behaviour of the material layers subjected to traffic loads. Many field cases and accelerated load testing (ALT) studies have shown that this assumption is reliable provided that the equivalent elastic properties for asphalt layers are properly selected according to temperature and load speed (e.g. Jameson and Hopman, 2000; Bodin et al., 2017). Tran et al. (2004) proposed a new approach based on the use of M4-5n belonging to the family of the Multi-Particle Model for Multilayer Materials (M4) (Chabot, 1997). The M4-5n advantageously reduces the initial problem by one dimension avoiding the explicit presence of the z-coordinate in the equations. This is an interesting feature for the numerical modelling (mesh generation and computation time). The constitutive behaviour assumed for this model is linear elastic. The consideration in this model of generalized stress fields which remain of finite values avoids the problem of singularity near cracks. M4-5n is particularly adapted to parametric studies (Chabot et al., 2013). This model considers five equilibrium equations per layer ( $n$ stands for the number of layer). In this model initially intended for the study of delamination in composite 
materials, the pavement is represented by a piling of plates of type M4-5n.

Recent advances of M4-5n in the domain of pavements have led to replace the modelling of soil originally of Boussinesq type by the use of a Winkler foundation (Berthemet and Chabot, 2013; Nasser and Chabot, 2017). These developments were implemented using the finite difference method for plane strain problems and result in shorter computation time. In this paper, we rather focus on the derivation of a systematic solution method of M4-5n based on the mixed FEM and intended for application to $3 \mathrm{D}$ problems. The starting point is the derivation of the variational principle based on the complementary energy theorem whose condition of statically admissible stress is taken into account using Lagrange multipliers. The mixed formulation is obtained by expressing stationarity of the Lagrangian with respect to all its fields. The identification of the Lagrange multipliers yields the generalized displacement fields of M4-5n. It is shown also that the functional obtained is equivalent to that of Hellinger-Reissner expressed for the generalized fields of M4-5n. Special attention is paid to the discretization process in order to avoid an ill-conditioned system of algebraic equations after discretization and to insure stability of the solution. The developed method is implemented in a FreeFem++ script.

This paper is outlined as follows: a reminder of the M4-5n strong formulation is given in section 1. Derivation of the mixed formulation is detailed in section 2 and discretization and solution by the mixed FEM is presented in section 3. Finally, the application of the developed method to analysis of a real pavement subject to an accelerated fatigue test is shown in section 4 .

\section{Review of the M4-5n strong formulation}

A comprehensive description of M4-5n has been provided in previous works, where the reader is referred for more information (Chabot, 1997; Naciri et al., 1998; Chabot and Ehrlacher, 1998). The purpose in this section is just to recall the basic equations of this model which are solved by a mixed FE method whose establishment is the topic of the present paper. Body forces are neglected and linear elasticity is assumed herein.

In the construction of M4-5n, the stress fields per layer are approximated by polynomials of the vertical $z$-direction. The mechanical generalized fields of M4-5n then only depend on coordinates $(x, y)$ of the horizontal plane normal to the $z$-direction.

In M4-5n, the usual stress tensor in each layer $i$ is replaced by the generalized stresses $M_{\alpha \beta}^{i}(x, y), Q_{\alpha}^{i}(x, y), N_{\alpha \beta}^{i}(x, y)(\alpha, \beta \in\{1,2\})$ which stand for components of the resultant of moment, the shear and the normal stress in the mid-plane of each layer, respectively. The related generalized displacements denoted $\Phi_{\alpha}^{i}(x, y), V^{i}(x, y)$, $\mathrm{U}_{\alpha}^{i}(x, y)$ stand for rotation, the vertical and the horizontal displacements in the same mid-plane locations, respectively. The connection between two consecutive layers, $i$ and $i+1$, is ensured by the interface shear stress, $\tau_{\alpha}^{i, i+1}(x, y)$, and the normal stress, $v^{i, i+1}(x, y)$. Each M4-5n layer has its own set of equations: equilibrium, compatibility, and elastic constitutive law. Considering summation over the repeated indices $\{\alpha, \beta\} \in$ $\{1,2\}$, these read within the considered domain $\Omega$ of plane $(x, y)$ :

For equilibrium within layer $i$,

$$
\begin{gathered}
\mathrm{N}_{\alpha \beta, \beta}^{\mathrm{i}}+\tau_{\alpha}^{\mathrm{i}, \mathrm{i}+1}-\tau_{\alpha}^{\mathrm{i}-1, \mathrm{i}}=0 \\
\mathrm{M}_{\alpha \beta, \beta}^{\mathrm{i}}-\mathrm{Q}_{\alpha}^{\mathrm{i}}+\frac{\mathrm{e}^{\mathrm{i}}}{2}\left(\tau_{\alpha}^{\mathrm{i}, \mathrm{i}+1}+\tau_{\alpha}^{\mathrm{i}-1, \mathrm{i}}\right)=0 \\
\mathrm{Q}_{\alpha, \alpha}^{\mathrm{i}}+v^{\mathrm{i}, \mathrm{i}+1}-v^{\mathrm{i}-1, \mathrm{i}}=0
\end{gathered}
$$


For equilibrium at interfaces $(0,1)$ and $(n, n+1)$,

$$
\begin{aligned}
& \tau_{1}^{0,1}=0 ; \tau_{2}^{0,1}=0 ; v^{0,1}=-q(x, y) \\
& \tau_{1}^{\mathrm{n}, \mathrm{n}+1}=0 ; \tau_{2}^{\mathrm{n}, \mathrm{n}+1}=0 ; v^{\mathrm{n}, \mathrm{n}+1}=0
\end{aligned}
$$

These equations take into account stresses imposed on the upper $(0,1)$ and the lower $(n, n+1)$ surface of the multilayer structure considered. In the present case, these are assumed equal to zero except for the vertical loading component $q(x, y)$ (considered positive downward).

For compatibility within layer $i$,

$$
\begin{gathered}
\varepsilon_{\alpha \beta}^{\mathrm{i}}=\frac{1}{2}\left(\mathrm{U}_{\alpha, \beta}^{\mathrm{i}}+\mathrm{U}_{\beta, \alpha}^{\mathrm{i}}\right) \\
\chi_{\alpha \beta}^{\mathrm{i}}=\frac{1}{2}\left(\Phi_{\alpha, \beta}^{\mathrm{i}}+\Phi_{\beta, \alpha}^{\mathrm{i}}\right) \\
\mathrm{d}_{\alpha}^{\mathrm{i}}=\Phi_{\alpha}^{\mathrm{i}}+\mathrm{V}_{, \alpha}^{\mathrm{i}}
\end{gathered}
$$

$\varepsilon^{i}$ is the tensor field of membrane strain, $\chi^{i}$ is the tensor field of curvature and $d^{i}$ denotes strain related to the resultant shear stress.

For compatibility at the interface between layers $i$ and $i+1$,

$$
\begin{gathered}
\mathrm{D}_{\alpha}^{\mathrm{i}, \mathrm{i}+1}=\mathrm{U}_{\alpha}^{\mathrm{i}+1}-\mathrm{U}_{\alpha}^{\mathrm{i}}-\frac{\mathrm{e}^{\mathrm{i}}}{2} \Phi_{\alpha}^{\mathrm{i}}-\frac{\mathrm{e}^{\mathrm{i}+1}}{2} \Phi_{\alpha}^{\mathrm{i}+1} \\
\mathrm{D}_{3}^{\mathrm{i}, \mathrm{i}+1}=\mathrm{V}^{\mathrm{i}+1}-\mathrm{V}^{\mathrm{i}}
\end{gathered}
$$

$D_{\alpha}$ and $D_{3}$ are the strains related to interface shear and pull out.

For the constitutive equations within layer $i$,

$$
\begin{aligned}
\varepsilon_{\alpha \beta}^{\mathrm{i}}= & \frac{1}{\mathrm{e}^{\mathrm{i}} \mathrm{E}^{\mathrm{i}}}\left(\left(1+v^{\mathrm{i}}\right) \mathrm{N}_{\alpha \beta}^{\mathrm{i}}-v^{\mathrm{i}} \mathrm{N}_{\mathrm{kk}} \delta_{\alpha \beta}\right) \text { for } \mathrm{k} \in\{1,2\} \\
\chi_{\alpha \beta}^{i}= & \frac{12}{e^{i} E^{i}}\left(\left(1+v^{i}\right) M_{\alpha \beta}^{i}-v^{i} M_{k k} \delta_{\alpha \beta}\right) \text { for } k \in\{1,2\} \\
& d_{\alpha}^{i}=\frac{12\left(1+v^{i}\right)}{5 e^{i} E^{i}} Q_{\alpha}^{i}-\frac{1+v^{i}}{5 E^{i}}\left(\tau_{\alpha}^{i-1, i}+\tau_{\alpha}^{i, i+1}\right)
\end{aligned}
$$

where $\delta$ is the Kronecker delta.

For the constitutive behaviour between layers $i$ and $i+1$,

$$
\begin{gathered}
D_{\alpha}^{i, i+1}=-\frac{1+v^{i}}{5 E^{i}} Q_{\alpha}^{i}-\frac{1+v^{i+1}}{5 E^{i+1}} Q_{\alpha}^{i+1}-\frac{e^{i}\left(1+v^{i}\right)}{15 E^{i}} \tau_{\alpha}^{i-1, i}-\frac{e^{i+1}\left(1+v^{i+1}\right)}{15 E^{i+1}} \tau_{\alpha}^{i+1, i+2}+ \\
\frac{4}{15}\left(\frac{e^{i}\left(1+v^{i}\right)}{E^{i}}+\frac{e^{i+1}\left(1+v^{i+1}\right)}{E^{i+1}}\right) \tau_{\alpha}^{i, i+1} \\
D_{3}^{i, i+1}=\frac{9 e^{i}}{70 E^{i}} v^{i-1, i}+\frac{9 e^{i+1}}{70 E^{i+1}} v^{i+1, i+2}+\frac{13}{35}\left(\frac{e^{i}}{E^{i}}+\frac{e^{i+1}}{E^{i+1}}\right) v^{i, i+1}
\end{gathered}
$$

In the above equations, $\mathrm{e}^{\mathrm{i}}$ and $\mathrm{E}^{\mathrm{i}}$ are the thickness and the Young modulus of layer i and $v^{i}$ is Poisson's ratio. The boundary conditions considered on $\partial \Omega$ in this paper are specified further.

\section{Taking into account cracks and debonding in M4-5n}

A crack is defined as crossing the whole thickness of one or several layers. As a consequence, the crack tip is always located at an interface and then does not change the in-layer equations defined on the mid-planes of the plates. In particular, the compatibility equations between the generalised displacement and strains remain valid. 
The introduction of a vertical crack is then performed by zeroing some of the generalized stresses along the crack contour projected on the $(x, y)$ plane, depending on the assumptions considered for load transfer (e.g. Eq. 10).

In addition, debonding of interface $(i, i+1)$ can be accounted for by zeroing $\tau_{\alpha}^{i, i+1}$ and also possibly $v^{i, i+1}$ on the involved area depending on the contact conditions considered in the vertical direction. These conditions result in discontinuities for the generalized fields of displacements and of interface shear and normal stress obtained from one side to the other of the contour of crack surfaces or delaminated areas.

\section{Derivation of a mixed formulation for M4-5n}

This section is focused on the establishment of a general and systematic method for solving the M4-5n equations based on the mixed FE method. This approach is natural in the sense that M4-5n is derived from the 3D Hellinger-Reissner (HR) functional (Reissner, 1951) and thus leads to energy densities expressed in terms of generalized stresses instead of generalized strains.

The starting point of the developed formulation is the 3D complementary energy theorem which states that among all statically admissible fields, $\mathcal{S}\left(T_{d}, f\right)$ ( $f$ denotes body force density), the stress field $\sigma$ which is the solution of the equilibrium problem in linear elasticity maximizes the complementary energy functional. For a solid represented by a volume $\Omega$ whose part of its boundary denoted $\partial \Omega_{U}$ is subject to the imposed displacements $u_{d}$ and the complementary part denoted $\partial \Omega_{T}$ is subject to the imposed stress $T_{d}$, this functional reads:

$$
\mathcal{F}\left(\sigma^{*}\right)=-\frac{1}{2} \int_{\Omega} \sigma^{*}: C: \sigma^{*} d \Omega+\int_{\partial \Omega_{U}}\left[\sigma^{*} \cdot n\right] \cdot u_{d} d S
$$

in which $\sigma^{*} \in \mathcal{S}\left(T_{d}, f\right)$ (piecewise continuous and piecewise continuously differentiable function), $C$ is the compliance tensor and $n$ is the outward normal to $\partial \Omega_{u}$. The first integral in the right hand side of Eq.7 is the opposite of the elastic energy expressed in terms of stress. Then the complementary energy theorem for $\mathcal{F}$ strictly convex simply reads:

$$
\text { For }(\sigma, u, \varepsilon) \text { solution, } \forall \sigma^{*} \in \mathcal{S}\left(T_{d}, f\right), \sigma^{*} \neq \sigma, \mathcal{F}\left(\sigma^{*}\right)<\mathcal{F}(\sigma)
$$

Then, the idea is to transpose this theorem to the generalized fields of M4-5n. Let us first present the density of elastic energy, $w_{e}$, for this model. $w_{e}$ is obtained from the elastic energy density derived from the 3D problem and considering few classical assumptions in particular related to the modelling of plates (Chabot, 1997). Thus the elastic energy density for M4-5n is a quadratic form of the generalized stress tensors and can be decomposed into three densities related to the several (symmetric) tensors. $w_{e}$ reads:

$$
\mathrm{w}_{e}=\sum_{i=1}^{n}\left(w_{c}^{i}+w_{v}^{i}+w_{Q}^{i}\right)
$$

where $w_{c}^{i}, w_{v}^{i}$ and $w_{Q}^{i}$ are given in the appendix.

For the sake of conciseness, we assume in the rest of the paper a combination of boundary conditions either traction free or zero displacement that is: 


$$
\begin{gathered}
N_{\alpha \beta}^{i} n_{\beta}=0 ; M_{\alpha \beta}^{i} n_{\beta}=0 ; Q_{\alpha}^{i} n_{\alpha}=0 \text { on } \partial \omega_{T}^{i} \\
U_{\alpha}^{i}=0 ; \Phi_{\alpha}^{i}=0 ; V^{i}=0 \text { on } \partial \omega_{U}^{i}
\end{gathered}
$$

Then in both bases the M4-5n complementary energy simply reads: $-\int_{\omega} w_{e} d \omega$, with $\omega$ the integration domain of M4-5n (2D or 1D). The maximum of this functional is sought for the generalized stress fields which are statically admissible. This condition can be accounted for using Lagrange multipliers denoted $\lambda^{i}$ and $\mu^{i}$. This results in the following functional (Lagrangian):

$$
\begin{aligned}
& L=-\int_{\omega} \mathrm{w}_{\mathrm{e}} \mathrm{d} \omega+\sum_{\mathrm{i}=1}^{\mathrm{n}} \int_{\omega}\left[\lambda_{\alpha}^{\mathrm{N}, \mathrm{i}}\left(\mathrm{N}_{\alpha \beta, \beta}^{\mathrm{i}}+\tau_{\alpha}^{\mathrm{i}, \mathrm{i}+1}-\tau_{\alpha}^{\mathrm{i}-1, \mathrm{i}}\right)+\lambda_{\alpha}^{\mathrm{M}, \mathrm{i}}\left(\mathrm{M}_{\alpha \beta, \beta}^{\mathrm{i}}-\mathrm{Q}_{\alpha}^{\mathrm{i}}+\right.\right. \\
& \left.\left.\frac{\mathrm{e}^{\mathrm{i}}}{2}\left(\tau_{\alpha}^{\mathrm{i}, \mathrm{i}+1}+\tau_{\alpha}^{\mathrm{i}-1, \mathrm{i}}\right)\right)+\lambda^{\mathrm{Q}, \mathrm{i}}\left(\mathrm{Q}_{\alpha, \alpha}^{\mathrm{i}}+v^{\mathrm{i}, \mathrm{i}+1}-v^{\mathrm{i}-1, \mathrm{i}}\right)\right] \mathrm{d} \omega+ \\
& \sum_{\mathrm{i}=1}^{\mathrm{n}} \int_{\partial \omega_{\mathrm{T}}^{\mathrm{i}}}\left[\mu_{\alpha}^{\mathrm{N}, \mathrm{i}}\left(\mathrm{N}_{\alpha \beta}^{\mathrm{i}} \mathrm{n}_{\beta}^{\mathrm{i}}-\overline{\mathrm{N}}_{\alpha}^{\mathrm{i}}\right)+\mu_{\alpha}^{\mathrm{M}, \mathrm{i}}\left(\mathrm{M}_{\alpha \beta}^{\mathrm{i}} \mathrm{n}_{\beta}^{\mathrm{i}}-\overline{\mathrm{M}}_{\alpha}^{\mathrm{i}}\right)+\mu^{\mathrm{Q}, \mathrm{i}}\left(\mathrm{Q}_{\alpha}^{\mathrm{i}} \mathrm{n}_{\alpha}-\overline{\mathrm{Q}}_{\alpha}^{\mathrm{i}}\right) \mathrm{ds}\right]
\end{aligned}
$$

In which quantities with an overbar denote imposed generalized stresses on $\partial \omega_{T}^{i}$ of normal $n$. These are taken equal to zero according to Eq.10. Note that the generalized stress fields involved in the Lagrangian above are not constrained anymore since the statically admissible condition has been relaxed. In the case of a plate geometry, $\omega$ is represented by a $2 \mathrm{D}$ surface. Imposed conditions at the upper and the lower surface of the multilayer under consideration involve the interface shear and normal stresses $\left(\tau_{\alpha}^{0,1}\right.$, $v^{0,1}, \tau_{\alpha}^{n, n+1}, v^{n, n+1}$ ) which must be replaced by their known values in the Lagrangian (integral over $\Omega$ ) according to Eq.2. Obviously, these quantities must not be considered as variables of the Lagrangian. Also we make the choice (explained further) of taking $\mu_{\alpha}^{N, i}, \mu_{\alpha}^{M, i}, \mu^{Q, i}$ equal to the opposite of functions $\lambda_{\alpha}^{N, i}, \lambda_{\alpha}^{M, i}, \lambda^{Q, i}$ on border $\partial \omega_{T}^{i}$. Then it can be shown successively that: (i) stationarity of the resulting expression with respect to the Lagrange multipliers yields the equilibrium equations within $\omega$; (ii) stationarity with respect to the values of the Lagrange multipliers on $\partial \omega_{T}^{i}$ leads to the traction free boundary conditions (Eq.10). In addition, the expression of $L$ can be further integrated by parts to avoid spatial derivatives of the generalized stress tensors and consider straightly differentiation with respect to the stress fields. After integration and because of the choice made concerning $\mu_{\alpha}^{N, i}, \mu_{\alpha}^{M, i}, \mu^{Q, i}$, integrals over $\partial \omega_{T}^{i}$ are found to remove from the expression of $L$ which now reads:

$$
\begin{gathered}
L=-\int_{\omega} \mathrm{w}_{e} d \omega+\sum_{i=1}^{n} \int_{\omega}\left[-\lambda_{\alpha, \beta}^{N, i} N_{\alpha \beta}^{i}+\lambda_{\alpha}^{N, i}\left(\tau_{\alpha}^{i, i+1}-\tau_{\alpha}^{i-1, i}\right)-\lambda_{\alpha, \beta}^{M, i} M_{\alpha \beta}^{i}+\right. \\
\left.\lambda_{\alpha}^{M, i}\left(-Q_{\alpha}^{i}+\frac{e^{i}}{2}\left(\tau_{\alpha}^{i, i+1}+\tau_{\alpha}^{i-1, i}\right)\right)-\lambda_{, \alpha}^{Q, i} Q_{\alpha}^{i}+\lambda^{Q, i}\left(v^{i, i+1}-v^{i-1, i}\right)\right] d \omega+ \\
\sum_{i=1}^{n} \int_{\partial \omega_{U}^{i}}\left[\lambda_{\alpha}^{N, i}\left(N_{\alpha \beta}^{i} n_{\beta}^{i}\right)+\lambda_{\alpha}^{M, i}\left(M_{\alpha \beta}^{i} n_{\beta}^{i}\right)+\lambda^{Q, i}\left(Q_{\alpha}^{i} n_{\alpha}\right)\right] d s
\end{gathered}
$$

Then taking equal to zero the variation of $L$ above with respect to the generalized stress fields (within layers and at interfaces) within $\omega$ leads to the relationships between the Lagrange multipliers and the generalized strain tensors associated to these stress fields through the M4-5n constitutive behaviour derived from the derivatives of the elastic energy density, $w_{e}$. Furthermore, stationarity of $L$ on $\partial \omega_{U}^{i}$ yields the boundary conditions for the Lagrange multipliers which happen to be zero on this border. Now setting: 


$$
\lambda_{\alpha}^{N, i}=-U_{\alpha}^{i} ; \lambda_{\alpha}^{M, i}=-\Phi_{\alpha}^{i} ; \lambda^{Q, i}=-V^{i}
$$

within the relationships obtained from stationarity of $L$ (in $\omega$ and on borders) after and prior to integration by parts allows us to verified the whole set of equations of the strong formulation (equilibrium, constitutive behaviour, kinematics compatibility, boundary conditions on $\partial \omega_{T}^{i}$ and $\partial \omega_{U}^{i}$ ). This attests of the equivalence between the strong formulation and the derived mixed formulation (without considering the question of the functional spaces on which each formulation is defined). Finally, substituting identification of the Lagrange multipliers (Eq.14) into Eq.13 leads to the following expression:

$$
\begin{gathered}
L=-\int_{\omega} w_{e} d \omega+\sum_{i=1}^{n} \int_{\omega}\left[U_{\alpha, \beta}^{i} N_{\alpha \beta}^{i}-U_{\alpha}^{i}\left(\tau_{\alpha}^{i, i+1}-\tau_{\alpha}^{i-1, i}\right)+\Phi_{\alpha, \beta}^{i} M_{\alpha \beta}^{i}-\right. \\
\left.\Phi_{\alpha}^{i}\left(-Q_{\alpha}^{i}+\frac{e^{i}}{2}\left(\tau_{\alpha}^{i, i+1}+\tau_{\alpha}^{i-1, i}\right)\right)+V_{, \alpha}^{i} Q_{\alpha}^{i}-V^{i}\left(v^{i, i+1}-v^{i-1, i}\right)\right] d \omega- \\
\sum_{i=1}^{n} \int_{\partial \omega_{U}^{i}}\left[U_{\alpha}^{i}\left(N_{\alpha \beta}^{i} n_{\beta}^{i}\right)+\Phi_{\alpha}^{i}\left(M_{\alpha \beta}^{i} n_{\beta}^{i}\right)+V^{i}\left(Q_{\alpha}^{i} n_{\alpha}\right)\right] d s
\end{gathered}
$$

which is equivalent to the HR functional expressed in terms of the generalized fields (under the arbitrary assumptions made on the boundary conditions Eq.(10) and Eq.(11) to simplify the mathematical expressions). The solution of the M4-5n problem is a saddle point for $L$ with regards to the generalized stress and displacement fields. To seek for this saddle point of $L$, Eq. 15 is differentiated with respect to all its arguments leading to equation $\delta L=0$ on which the FE solution of M4-5n relies.

\section{Discretization and solution of the mixed formulation}

The solution of the mixed formulation obtained in the previous section is approximated using the FE method with its classical rules to derive a discretized problem from the weak formulation given by Eq.(15). The solution procedure is implemented using the FreeFem ++ (Hecht, 2012) environment which handles discretization of domain $\Omega$ and of the generalized field variables automatically provided that the mesh and the associated interpolation spaces (of finite dimensions) are defined. The generalized fields which do not involve derivatives in Eq.(15) (basically the stress fields that we gather all together under notation $\Sigma$ ) must be at least of type $\mathcal{C}^{-1}$ (piecewise constant). On the other hand, the generalized displacement fields, denoted $\mathcal{U}$ as a whole, involving first order derivatives must be at least of type $\mathcal{C}^{0}$ (piecewise continuous). Because of the layer-wise construction of M4-5n, the elementary "stiffness" matrices resulting from discretization are banded since no interaction occurs between layers $i$ and $i+2$. Given the structure of the Lagrangian under consideration (sum of quadratic and linear forms) the linear system to be solved once the assembly process has been performed reads:

$$
\left[\begin{array}{cc}
-\left[K_{\Sigma \Sigma}\right] & {\left[K_{\Sigma \mathrm{U}}\right]} \\
{\left[K_{\mathrm{U} \Sigma}\right]} & 0
\end{array}\right]\left(\begin{array}{l}
\{\Sigma\} \\
\{U\}
\end{array}\right)=\left(\begin{array}{l}
\left\{\mathrm{F}_{\Sigma}\right\} \\
\left\{F_{U}\right\}
\end{array}\right)
$$

which is a classical form when it comes to mixed formulation (Zienkiewicz and Taylor, 2000; Bathe, 2006). $\{\Sigma\}$ and $\{U\}$ are the nodal arrays of the generalized stress and displacement fields, respectively. $\left[K_{\Sigma \Sigma}\right]$ is a symmetric positive definite compliance matrix and $\left[K_{U \Sigma}\right]=\left[K_{\Sigma U}^{T}\right]$. The whole matrix is symmetric and banded. $\left\{F_{\Sigma}\right\}$ is the right 
hand side array related to imposed stress at the surface of the multilayer structure brought in through the elastic energy and to the imposed displacements on $\partial \omega_{U}$ (if not equal to zero). $\left\{F_{U}\right\}$ is the right hand side array related to the imposed stress at the surface of the multilayer structure brought in through the equilibrium equations or $\partial \Omega_{T}$.

This kind of algebraic system with zeros on part of the diagonal is known to be potentially over- or under-determined depending on the interpolation spaces of $\{\Sigma\}$ (resp. $\{\delta \Sigma\}$ ) and $\{U\}$ (resp. $\{\delta U\}$ ). A first condition to avoid the under-determination issue consists in picking out the dimension of the Lagrange multiplier space (denoted $n_{U}$ for $\{U\}$ ) constraining the problem lower than that of the primal quantities (denoted $n_{\Sigma}$ for $\left.\{\Sigma\}\right): n_{U}<n_{\Sigma}$. Another condition to be verified to ensure unicity of the solution is the invertibility of $K_{\Sigma U}$. These two requirements yield the so-called LadyzhenskayaBabuška-Breezi (LBB) conditions which are necessary and sufficient to ensure existence and unicity of mixed formulations. While the first condition is easy to achieve a priori, the second will be checked a posteriori based on the well-progress of the solution process.

We implemented two different choices of functional spaces (verifying the LBB conditions) in the Freefem++ scripts developed to compute the solution. The first one relies on Lagrange polynomials in $x$ and $y$ of degree relatively high whose order is function of the type of fields addressed. We have made the following choices:

$$
\begin{array}{cc}
\operatorname{order}\left(P_{N_{\alpha \beta}^{i}}\right)=3, \operatorname{dim}\left(P_{N_{\alpha \beta}^{i}}\right)=10 ; & \operatorname{order}\left(P_{M_{\alpha \beta}^{i}}\right)=2, \operatorname{dim}\left(P_{M_{\alpha \beta}^{i}}\right)=6 \\
\operatorname{order}\left(P_{Q_{\alpha}^{i}}\right)=3, \operatorname{dim}\left(P_{Q_{\alpha}^{i}}\right)=10 ; & \operatorname{order}\left(P_{\tau_{\alpha}^{i}}\right)=2, \operatorname{dim}\left(P_{\tau_{\alpha}^{i}}\right)=6 \\
\operatorname{order}\left(P_{v^{i, i+1}}\right)=2, \operatorname{dim}\left(P_{v^{i, i+1}}\right)=6 ; & \operatorname{order}\left(P_{U_{\alpha}^{i}}\right)=3, \operatorname{dim}\left(P_{U_{\alpha}^{i}}\right)=10 \\
\operatorname{order}\left(P_{\Phi_{\alpha}^{i}}\right)=1, \operatorname{dim}\left(P_{\Phi_{\alpha}^{i}}\right)=3 ; & \operatorname{order}\left(P_{V^{i}}\right)=2, \operatorname{dim}\left(P_{V^{i}}\right)=6
\end{array}
$$

in which the dimensions of spaces are given by $\operatorname{dim}=($ order +1$)(\operatorname{order}+2) / 2)$ that is the number of monomials in the Lagrange polynomials. In particular, this choice leads to the triangular finite element shown in Figure 1 and used in the Freefem++ meshes. Its elementary stiffness matrix for $n$ layers is of size $118 n-12 \times 118 n-12$. The number of degrees of freedom (DOF) becomes rapidly great for meshes composed of such elements. However, as a benefit coarse meshes can be considered when using this type of element because of its high level of accuracy. Note also that the elementary matrix is of great size but it has many zeros due to its property of bandedness.

The second choice of interpolation involves the so-called P1-bubble element for fields $\Sigma$ and P1 (linear interpolation) for fields $U$. The P1-bubble element consists in adding to the P1 element one DOF at the barycentre of the element. This DOF is associated to the following interpolation function with bubble shape:

$$
N_{b}\left(b_{1}, b_{2}, b_{3}\right)=\frac{b_{1} b_{2} b_{3}}{27}
$$

where $b_{1}, b_{2}$ and $b_{3}$ are the barycentric coordinates of the point considered within the triangular element. This function is equal to 1 at the barycentre and to 0 on edges of the element. Then, the stress components of $\Sigma$ are interpolated as follows:

$$
\Sigma(\mathrm{x}, \mathrm{y})=\sum_{\mathrm{i}=1}^{3} \mathrm{~N}_{\mathrm{i}}(\mathrm{x}, \mathrm{y}) \Sigma_{\mathrm{i}}+\mathrm{N}_{\mathrm{b}}\left(\mathrm{b}_{1}(\mathrm{x}, \mathrm{y}), \mathrm{b}_{2}(\mathrm{x}, \mathrm{y}), \mathrm{b}_{3}(\mathrm{x}, \mathrm{y})\right)\left(\Sigma_{\mathrm{b}}-\frac{\Sigma_{1}+\Sigma_{2}+\Sigma_{3}}{3}\right)
$$

in which $N_{i}$ are the shape functions of the P1 element and $\Sigma_{b}$ is the value of $\Sigma$ at the 
barycentre. The M4-5n elementary matrix for this type of triangular element P1bubble/P1 is of size $59 n-8 \times 59 n-8$, i.e. approximately four times less than for the previous choice. Note that this size can be diminished to that of an element of type P1/P1 $(48 n-6 \times 48 n-6)$ by means of static condensation (loss of the bandedness property).

Once the system has been assembled on the basis of the elementary matrices deriving from one or the other of the two choices described above, the linear system (Eq.16) is solved according to a multifrontal Gauss LU factorization (for sparse matrices).

The validation of the mixed FE method and its implementation was performed by comparison to prior M4-5n solutions (finite differences) developed in plane strain (leading to a 1D discretization in the frame of M4-5n) (Nasser et al., 2016). An analytical solution was also used for the validation (Nasser, 2016). Application of the method developed on a M4-5n 3D case (2D discretization) is presented in section 5.

\section{Improvements to the M4-5n mixed formulation}

In this section, we present some adjustments to the M4-5n formulation that are useful in applications dedicated to the analysis of pavements.

\section{Elastic support for M4-5n}

To be more representative of the soil behaviour of a pavement, a Winkler support can be added to the M4-5n plates. Considering this condition leads to two modifications to be accounted for in the formulation: the normal stress $v^{n, n+1}$ below the bottom layer becomes an unknown variable and is taken into account through the elastic energy density of Winkler springs expressed, in terms of stress, as follows:

$$
\mathrm{W}_{\text {winkler }}=\frac{1}{2} \frac{\left(v^{n, n+1}\right)^{2}}{k}
$$

where $k$ is the stiffness of Winkler springs. In the variational form, $w_{\text {winkler }}$ must be added to $w_{e}$.

\section{Enforcing the stress boundary conditions}

As explained in section 2, the stress boundary conditions are imposed through Lagrange multipliers $\mu^{i}$ in the variational formulation. These are assumed to be equal to $-\lambda^{i}$ on $\partial \omega_{T}^{i}$. However, once discretization has been performed this relationship between values of the Lagrange multipliers in $\Omega$ and at its boundary does not allow us to strictly reach the condition. Then, to enforce the stress boundary conditions in the discrete form of the problem, we add the following penalty term to the Lagrangian:

$$
-\frac{1}{2} \sum_{\mathrm{i}=1}^{\mathrm{n}} \int_{\partial \omega_{\mathrm{T}}^{\mathrm{i}}}\left[\Lambda_{\mathrm{N}}^{\mathrm{i}}\left(\mathrm{N}_{\alpha \beta}^{\mathrm{i}} \mathrm{n}_{\beta} \mathrm{N}_{\alpha \gamma}^{\mathrm{i}} \mathrm{n}_{\gamma}\right)+\Lambda_{\mathrm{M}}^{\mathrm{i}}\left(\mathrm{M}_{\alpha \beta}^{\mathrm{i}} \mathrm{n}_{\beta} \mathrm{M}_{\alpha \gamma}^{\mathrm{i}} \mathrm{n}_{\gamma}\right)+\Lambda_{\mathrm{Q}}^{\mathrm{i}}\left(\mathrm{Q}_{\alpha}^{\mathrm{i}} \mathrm{n}_{\alpha} \mathrm{Q}_{\beta}^{\mathrm{i}} \mathrm{n}_{\beta}\right)\right] \mathrm{d} \mathrm{s}
$$

in which $\Lambda_{N}^{i}, \Lambda_{M}^{i}, \Lambda_{Q}^{i}$ are arbitrary penalty coefficients, positive and of great values chosen a priori but in relation with the order of magnitude of the solution for $N, M$, and 
$Q$, respectively. Addition of this penalty term in the variational formulation yields the augmented Lagrangian.

\section{Accounting for vertical cracks and interface debonding}

This section deals with two types of discontinuity (of zero thickness): discontinuities within vertical plans (joints or cracks) and discontinuities at interfaces (construction will, partial debonding due to damaging at the interface between layers).

To take into account a vertical crack spreading over the height of one or several layers, the developed approach requires the insertion, at the location of the crack, of a vertical plan in the mesh in which nodes are doubled to enable jumps of discontinuous fields. Then, for the cracked layers of the structure the traction free boundary condition applies and is handled by penalization according to Eq.21. For the non-cracked layers of this plan, the generalized displacements and stresses are made continuous by penalization two-by-two of the fields face-to-face by adding terms as:

$$
-\frac{\Lambda}{2} \sum_{\mathrm{i}=1}^{\mathrm{n}_{\mathrm{nf}}} \int_{\Gamma_{\mathrm{f}}}\left[\begin{array}{r}
\left(\mathrm{U}_{\alpha}^{\mathrm{i}+}-\mathrm{U}_{\alpha}^{\mathrm{i}-}\right)\left(\mathrm{U}_{\alpha}^{\mathrm{i}+}-\mathrm{U}_{\alpha}^{\mathrm{i}-}\right)+\left(\phi_{\alpha}^{\mathrm{i}+}-\phi_{\alpha}^{\mathrm{i}-}\right)\left(\phi_{\alpha}^{\mathrm{i}+}-\phi_{\alpha}^{\mathrm{i}-}\right) \\
+\left(\mathrm{V}^{\mathrm{i}+}-\mathrm{V}^{\mathrm{i}-}\right)\left(\mathrm{V}^{\mathrm{i}+}-\mathrm{V}^{\mathrm{i}-}\right)
\end{array}\right] \mathrm{ds}
$$

Or

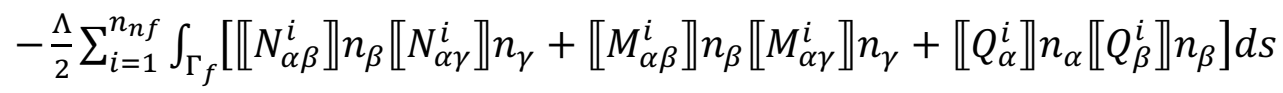

with $n_{n f}$ the number of non-cracked layers. $\Gamma_{f}$ is the trace of the vertical crack plan over plan $(0, x, y),(\cdot)^{+}$et $(\cdot)^{-}$denote quantities from both sides of $\Gamma_{f}$ and $\llbracket \cdot \rrbracket$ is the jump $(\cdot)^{+}-(\cdot)^{-}$. Existing discontinuities at ends of a crack related to $\tau_{\alpha}^{i, i+1}$ and $v^{i, i+1}$ are made possible by doubling the interface DOF's.

In the general case for which the crack tip does not coincide with a physical interface of the multilayer structure, the layer that contains the crack can be split in two sublayers of similar mechanical properties to enable application of the technique described right above. This cutting may also introduce non horizontal interfaces which can be dealt with varying the thickness $e^{i}(x, y)$ of the sublayers in the Lagrangian formulation.

About debonding, we assume that debonded faces remain in contact but may slip without friction. In this case, the interface shear stress $\tau_{\alpha}^{i, i+1}$ only is zero. This condition can be straightly enforced through penalization by adding for interface $(i, i+1)$ the following term:

$$
-\frac{\Lambda}{2} \int_{D} \tau_{\alpha}^{\mathrm{i}, \mathrm{i}+1} \tau_{\alpha}^{\mathrm{i}, \mathrm{i}+1} \mathrm{~d} \omega
$$

in which $D$ is the projection of the debonded domain over plan $(x, y)$. Continuity of the vertical displacements at the interface is naturally verified by construction of M4-5n as long as no specific condition is imposed on the interface normal stress $v^{i, i+1}$. However, nodes must be doubled on $\partial D$ to account for the discontinuity of $\tau_{\alpha}^{i, i+1}$ on this boundary. Continuity must then be reestablished for the other fields following the penalization technique used to derive Eq.23.

Two remarks can be made to close this section: 
(1) The penalty methods described above are not necessary for vertical cracks located on a symmetry plan of the mesh. In this case, cracks are taken into account through the boundary conditions of zero traction vector or zero displacement (possibly mixed) as expressed in section 2 .

(2) The expression of the augmented Lagrangian can usefully be made dimensionless with all the aforementioned fields having the same order of magnitude. This makes it possible to choose a unique value a priori for all the penalty coefficients independently of the nature of the coefficient to be penalized.

These improvements to the method developed originally were implemented in the Freefem++ scripts mentioned in section 3.

\section{Application to the study of a real pavement ALT testing}

In this section, the developed method implemented using the Freefem++ environment is applied to the study of a cracked real bituminous pavement which was instrumented and subjected to traffic using the so-called FABAC accelerated load testing facility (see Figure 2) (Perez et al., 2007). The aim of this test was to follow the propagation of cracking in the pavement. To localize the initiation of damage in the structure, a transversal metal corner of $3 \mathrm{~cm}$ height was placed at the bottom of the asphalt layers. As a matter of fact a transversal crack was observed at the pavement surface right above the metal corner after approximately one million loading cycles. The M4-5n modelling presented further is related to this specific bottom-up cracking.

At different stages of this experiment, Falling Weight Deflectometer (FWD) campaigns were run to evaluate the evolution of deflection with damaging of the structure. These monitoring sessions took place prior to launching the fatigue experiment (FWD0) and at the end of it, as well as after 350000 loading cycles (FWD1). The applied FWD load was equal to $65 \mathrm{kN}$ with an "equivalent frequency" considered of about $35 \mathrm{~Hz}$. For a given FWD campaign, the different measurements are performed at small distances $(10 \mathrm{~cm})$ between each other one stepping over the metal corner location (in $x=0$ in Figure 2). Simulations of these tests with M4-5n are shown below.

The structure under consideration is composed of two HMA layers of height 5 and $6 \mathrm{~cm}$, respectively. These are resting on a granular base and soil. The mechanical characteristics of the HMA are known from complex modulus tests performed in the laboratory. Since M4-5n is developed in elasticity, an equivalent elastic modulus for the HMA materials under FWD loading is needed for the computations. This is obtained from the Huet-Sayegh model (Huet, 1963, 1999; Sayegh, 1965) fitted from the complex modulus tests and is taken equal to the norm of the complex modulus computed for the FWD "equivalent frequency" and the temperature within the HMA layers measured during the FWD campaign (about $12^{\circ} \mathrm{C}$ ). The equivalent elastic moduli obtained are equal to $14000 \mathrm{MPa}$.

\section{Modelling of the FWD campaigns (no inertia forces)}

The pavement tested is modelled as a bilayer M4-5n structure resting on a Winkler foundation representing both the granular base and soil. This structure is assumed homogeneous apart from cracks and its total thickness is set to $11 \mathrm{~cm}$. Following the 
rules defined in Section 4.3, the interface between the two layers is positioned according to the crack tip location and does not coincide necessarily with the physical interface between the asphalt layers.

Inertia forces are neglected and the FWD loading is modelled as a static force with intensity equal to the maximum value $\left(F_{\max }\right)$ obtained during the FWD rebound. Then the conditions at the pavement surface read: $\tau_{\alpha}^{0,1}=0, v^{0,1}=-q$ under the FWD load and $v^{0,1}=0$ elsewhere, $\tau_{\alpha}^{2,3}=0$ with $q=F_{\text {max }} / S$ and $S$ denoting the FWD imprint area. In practice, we take $q=0.72 \mathrm{MPa}$ applied on a square of side $30 \mathrm{~cm}$ (resultant equal to $65 \mathrm{kN}$ ). In our computations, we move this load step-by-step from 0 to $1 \mathrm{~m}$ every $5 \mathrm{~cm}$. As an illustration, Figure 3 shows two meshes of the problem corresponding to two locations of the FWD load.

Only quarter of the structure is modelled because of symmetry with respect to $(O, x, z)$ and $(O, y, z)$. Then the following boundary conditions are applied on the four borders of the two layers numbered from 1 to 4 as shown in Figure 2:

- Border \#1: $U_{2}^{i}(x, 0)=0, \Phi_{2}^{i}(x, 0)=0, Q_{2}^{i}(x, 0)=0$ (symmetry with respect to $(0, x, z))$

- Border \#2: $U_{\alpha}^{i}\left(L_{x} / 2, y\right)=0, \Phi_{\alpha}^{i}\left(L_{x} / 2, y\right)=0, V^{i}\left(L_{x} / 2, y\right)=0$ (clamped boundary condition)

- Border \#3: $U_{\alpha}^{i}\left(x, L_{y} / 2\right)=0, \Phi_{\alpha}^{i}\left(x, L_{y} / 2\right)=0, V^{i}\left(x, L_{y} / 2\right)=0$ (clamped boundary condition)

- Border \#4: when the location of the load centre is not in $x=0$ two computations are obviously needed to represent the loading according to the symmetrized geometry. The ad hoc load magnitude must be considered for each computation. In the first computation, the following symmetry conditions with respect to $(O, y, z)$ are imposed: $U_{1}^{i}(x, 0)=0, \Phi_{1}^{i}(x, 0)=0, Q_{1}^{i}(x, 0)=0$. In the second computation, the following anti-symmetry conditions with respect to $(0, y, z)$ are imposed: $N_{11}^{i}(0, y)=0, \mathrm{M}_{11}^{i}(0, y)=0, V^{i}(0, y)=0$. Taking advantage of the linearity of the M4-5n equations, the solution is obtained by summation of the solutions stemming from the two computations. When the load is in $x=0$ only one computation with the symmetry conditions is needed.

Campaigns FWD0 and FWD1 are simulated. FWD0 was performed on the healthy pavement so the interface for this campaign is positioned coincident with the physical one between the two HMA layers. About FWD1, two scenarios are envisaged to interpret the measurements obtained after 350000 loadings. In scenario \#1 the bilayer structure is assumed cracked over its whole transversal length in the symmetry plane $x=0$. The crack height $\left(h_{f}\right)$ is not measured directly. It is only estimated based on the results from the FWD1 campaign (after 350000 ALT loading cycles) and the $3 \mathrm{~cm}$ height metal corner placed at the bottom of the asphalt layers $\left(h_{f}=3\right.$ and $\left.5 \mathrm{~cm}\right)$. This is modelled by setting the thickness of the lower layer to $h_{f}$ and summing Eq.21 to the Lagrangian. In scenario \#2, the geometry of scenario \#1 is complemented by a vertical crack lying on the whole length of the structure along the symmetry plane $y=0$. This crack is also assumed of height $h_{f}$. The results from the FWD campaigns are analysed plotting the maximum deflection obtained under the falling weight when the latter is moved step-by-step from $x=0$ (location of the defect in the x-direction) to $x=0.5 \mathrm{~m}$ (Figure 4).

The spring stiffness of the Winkler foundation is back calculated from the value of deflection stemming from FWD0 and is set to $k=150 \mathrm{MPa} / \mathrm{m}$. This value leads to a 
numerical deflection of $452 \mu m$ for the healthy structure which after conversion using Odemark's formula (Odemark, 1949) corresponds to a homogeneous semi-infinite platform of modulus $75 \mathrm{MPa}$ (in good agreement with that measured on site prior to the asphalt concrete laying). Figure 4 shows the experimental measurements from FWD0 and FWD1 as well as the simulations performed for a non-cracked structure and those following scenario \#1 and \#2. As expected, simulation by M4-5n of the healthy structure leads to a constant deflection with $x$, which is close to that measured during FWD0. Measurements obtained after 350000 FABAC loading (FWD1) show two features departing from FWD0: (i) a global increase of about $50 \mu \mathrm{m}$ in the circulated area of the FABAC machine, (ii) a hat-shaped deflection curve above the initial defect placed in the structure. To retrieve these effects by means of simulation we first tried to interpret them through a transversal crack which would have developed in the vertical plane of the defect (scenario \#1). Two heights of crack were tested: $h_{f}=0.03 \mathrm{~m}$ and $h_{f}=0.05 \mathrm{~m}$. The results from these computations are also plotted in Figure 4. The hatshaped trend is indeed highlighted considering this scenario. For $h_{f}=0.05 \mathrm{~m}$, the maximum value of the deflection computed is equal to that measured. However this scenario does not account for the overall increase of the deflection level. A first possible explanation could be a change of the bearing capacity of soil due to an increase of water content between FWD0 and FWD1. It should be possible to check this at the end of the experiment after dismantling the pavement. Other causes are possible to explain these measurements (temperature change; Nasser, 2016). Here we examine another possible explanation (scenario \#2) which illustrates the capability of the developed mixed FEM program to handle crossing of two vertical cracks. Indeed as shown in Figure 4, scenario \#2 can lead to both a hat-shaped curve and an overall increase of deflection. Cracks of height $h_{f}=0.05 \mathrm{~m}$ lead to overestimation of the deflection but those of height $h_{f}=0.03 m$ yield a good estimate of the measurements.

The post-mortem analysis of the structure planned after completion of the FABAC test will include trenches and should help identify the actual origin for the change of the pavement response with time. The major point emerging from applying M4-5n to the present study of a real pavement is that the mixed FEM combined to M45 n offers a promising numerical tool for investigation of various cracking scenarios in pavements.

\section{Conclusion}

We have presented in this paper the development of a numerical tool dedicated to the analysis of 3D pavement structures assumed elastic and with cracks or debonding areas. This tool relies on a model of plate-type called M4-5n. We have generalized the solving procedure of the model by use of the mixed finite element method. The solution procedure was implemented into a Freefem ++ script and validated against plane strain problems and an analytical solution of a plate bending problem. As an illustration, the developed method was used to simulate the response of a real pavement structure tested under accelerated loading conditions for which two scenarios of cracking were examined. Two main benefits are expected from this novel approach. One is the simplification on how taking into account complex crack geometry as compared to classical 3D FEM models. The other is related to the resulting finite values of the generalized interface stresses that can be used straightforward in criteria for crack growth. 


\section{References}

Bathe, K.J. (2006). Finite Element Procedures.

Batoz, J.L. (1995). Modélisation des structures par éléments finis -Tome 1. Hermès Lavoisier.

Berthemet, F., \& Chabot, A. (2013). Apports du massif de Winkler dans la construction d'un outil de calcul de structure multicouche fissurée reposant sur un sol. 21ème Congrès Français de Mécanique. August 26-30, Bordeaux. Retrieved from http://documents.irevues.inist.fr/bitstream/handle/2042/52168/a_69E3H71P.pdf? sequence $=1$

Bodin, D., Chupin, O., \& Denneman, E. (2017). Viscoelastic pavement simulations and equivalent asphalt modulus. Journal of Testing and Evaluation, 45(6), 18871895. doi:10.1520/JTE20160652

Chabot, A. (1997). Analyse des efforts à l'interface entre les couches des matériaux composites à l'aide de Modélisations Multiparticulaires des Matériaux Multicouches (M4). ENPC PhD thesis, Paris. Retrieved from https://tel.archivesouvertes.fr/tel-00197853

Chabot, A., \& Ehrlacher A. (1998). Modèles Multiparticulaires des Matériaux Multicouches M4_5n et M4_(2n+1)M pour l'étude des effets de bord. Proceedings of the Comptes-rendus aux 11ème Journées Nationales sur les Composites (JNC11), 3, 1389-1397, Nov. 18-20, Arcachon, France. Retrieved from https://hal.archives-ouvertes.fr/hal-00325238/file/JNC11.pdf

Chabot, A., Hun, M., \& Hammoum, F. (2013). Mechanical analysis of a mixed mode debonding test for composite pavements. Construction and Building Materials 40:1076-1087

Chabot, A., Buttlar, B., Dave, E., Petit, C., \& Tebaldi, G. (Eds), 1st ed. (2016). 8th RILEM International Conference on Mechanisms of Cracking and Debonding in Pavements. Springer Series: RILEM Bookseries, Vol. 13, ISBN978-94-0240866-9. doi: 10.1007/978-94-024-0867-6

Hecht, F. (2012). New development in FreeFem++. Journal of Numerical Mathematics 20, no. 3-4, 251-265. 65Y15. doi 10.1515/jnum-2012-0013

Huet, C. (1963). Etude par une méthode d'impédance du comportement viscoélastique des matériaux hydrocarbonés. PhD Thesis. Faculty of Sciences of Université de Paris, France.

Huet, C. (1999). Coupled size and boundary-condition effects in viscoelastic heterogeneous and composite bodies. Mechanics of Materials 1999; 31: 787829.

Jameson, G., \& Hopman, P. (2000). Austroads Pavement Design Guide Chapter 6: 23 Development of Relationships between Laboratory Loading Rates and Traffic Speed. ARRB, Page 20 of 241 Vermont South, Australia.

Naciri, T., Ehrlacher, A., \& Chabot, A. (1998). Interlaminar Stress Analysis with a new Multiparticle Modelisation of Multilayered Materials (M4).Composites Sciences and Technology, 58 (3), 337-343. doi: 10.1016/S0266-3538(97)00085-7

Nasser, H., Piau, J.M., Chupin, O., \& Chabot, A. (2016). M4-5n numerical solution using the Mixed FEM, validation against the Finite Difference Method. 8th International conference on Mechanisms of Cracking and Debonding in Pavements (MCD2016), Nantes, France, June 7-9, Springer RILEM Bookseries, 13: 363-369, ISBN: 978-94-024-0867-6. doi: 10.1007/978-94-024-0867-6_51.

Nasser, H. (2016). Solving M4-5n by a Mixed Finite Element method for the analysis of pavements with discontinuities. ECN PhD thesis, Université Bretagne Loire, 2016. Retrieved from https://hal.archives-ouvertes.fr/tel-01529735. 
Nasser, H., \& Chabot, A. (2017). A Half-analytical Elastic Solution for 2D Analysis of Cracked Pavements. Advances in Engineering Software (In press) - doi: 10.1016/j.advengsoft.2017.06.008

Odemark, N. (1949). Investigations as to the elastic properties of soils and design of pavements according to the theory of elasticity. Report 77, National Swedish Road and Traffic Research Institute, Linkoping, Sweden.

Perez, S.A., Balay, J.M., Tamagny, P., \& Petit, C. (2007). Accelerated pavement testing and modeling of reflective cracking in pavements. Engineering Failure Analysis, 14: $1526-1537$.

Pommier, S., Gravouil, A., Moës, N., \& Combescure, A. (2009). La simulation numérique de la propagation des fissures : milieux tridimensionnels, fonctions de niveau, éléments finis étendus et critères énergétiques. Hermes - Lavoisier.

Reissner, E. (1950). On a Variational Theorem in Elasticity. Journal of Mathematics and Physics, 29: 90-95.

Sayegh, G. (1965). Contribution à l'étude des propriétés viscoélastiques des bitumes purs et des bétons bitumineux. PhD Thesis. Faculty of Sciences of Université de Paris, France.

Tran, Q. D., Chabot, A., Ehrlacher, A., \& Tamagny, P. (2004). A simplified modelling for cracking in pavements. Fifth International RILEM Conference Cracking in Pavements, May 5-8, Limoges, France. In Rilem Proceedings PRO 37, 299-306, Print ISBN 2-912143-47-0.

Zienkiewicz, O.C., \& Taylor, R.L. (2000). Finite Element Method, Vol. 1 - The Basis. Butterworth-Heinemann, Oxford, $5^{\text {th }}$ edition edition. ISBN : 978-0-7506-5049-6.

\section{Figures}

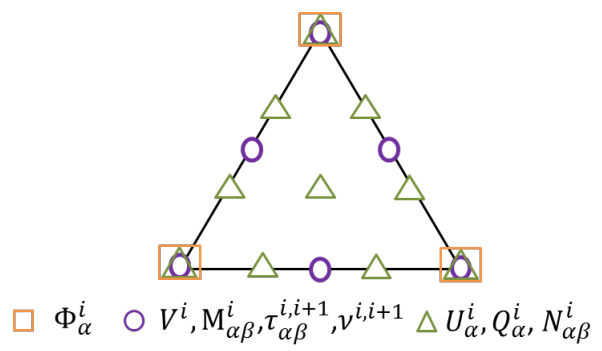

Figure 1. M4-5n mixed finite element with multiple nodes related to the field interpolations and of multiple degrees of freedom related to the number of layers in the structure

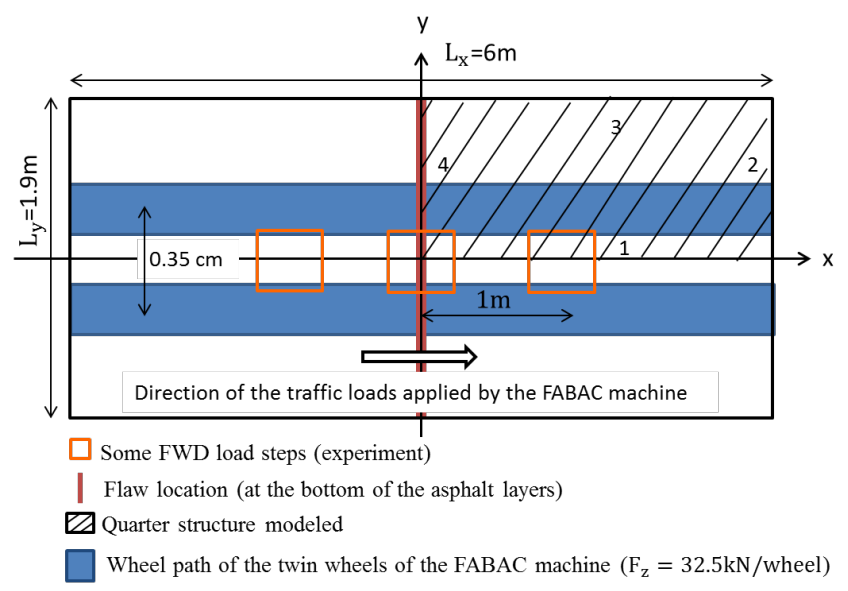


Figure 2. Sketch of the top view of the pavement tested by the FABAC machine and some locations of FWD tests performed as part of the experimental campaigns

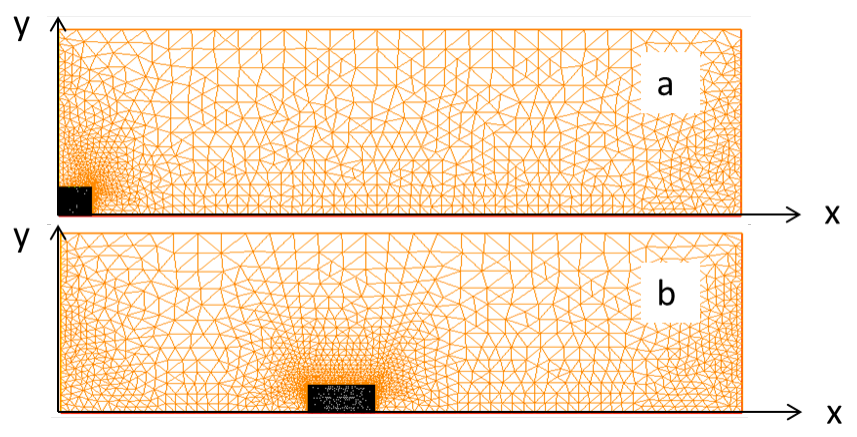

Figure 3. Meshes of a quarter of the pavement structure for two locations of the FWD load

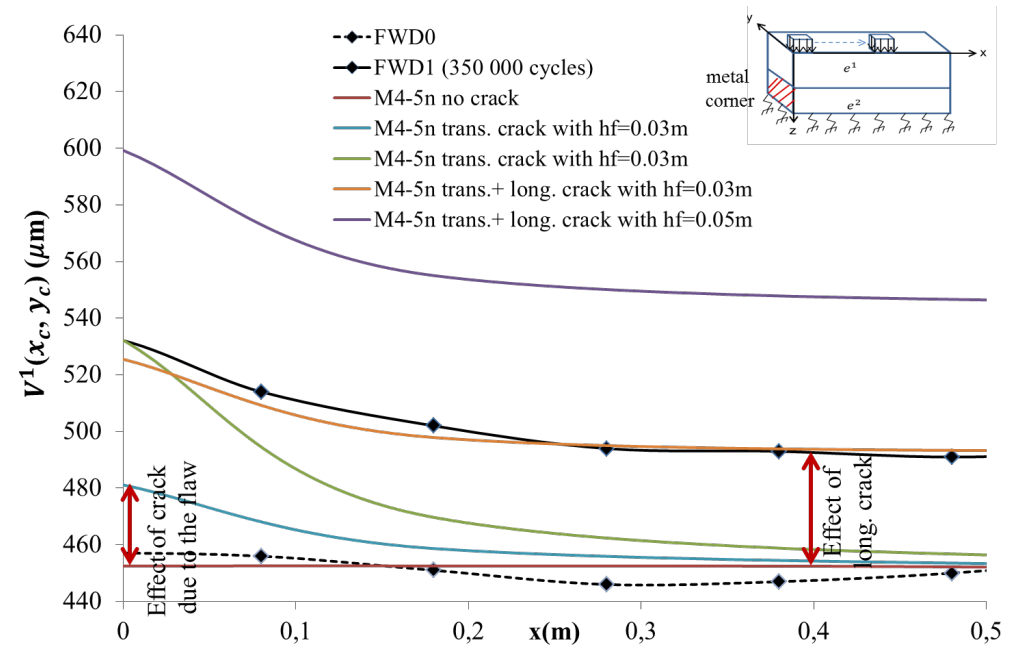

Figure 4. Deflection under the falling weight as a function of its location: experimental results and simulations related to scenario\#1 and scenario\#2 for two heights of cracking

\section{Appendix}

The elastic energy density for M4-5n is decomposed into three densities: $w_{c}^{i}, w_{v}^{i}$ and $\mathrm{w}_{\mathrm{Q}}^{\mathrm{i}}$ given as follows:

$$
\begin{gathered}
\mathrm{w}_{v}^{\mathrm{i}}=\frac{1}{2}\left[\frac{13 \mathrm{e}^{\mathrm{i}}}{35 \mathrm{E}^{\mathrm{i}}}\left(v^{\mathrm{i}, \mathrm{i}+1^{2}}+v^{\mathrm{i}-1, \mathrm{i}^{2}}\right)+\frac{9 \mathrm{e}^{\mathrm{i}}}{35 \mathrm{E}^{\mathrm{i}}} v^{\mathrm{i}, \mathrm{i}+1} v^{\mathrm{i}-1, \mathrm{i}}\right] \\
\mathrm{w}_{\mathrm{Q}}^{\mathrm{i}}= \\
\frac{1}{2}\left[\begin{array}{c}
\frac{12\left(1+v^{i}\right)}{5 \mathrm{e}^{\mathrm{i}} \mathrm{E}^{\mathrm{i}}}\left(Q_{1}^{i^{2}}+Q_{2}^{i^{2}}\right)+\frac{4 \mathrm{e}^{\mathrm{i}}\left(1+v^{i}\right)}{15 \mathrm{E}^{\mathrm{i}}}\left(\tau_{1}^{\mathrm{i}, \mathrm{i}+1^{2}}+\tau_{1}^{\mathrm{i}-1, \mathrm{i}^{2}}+\tau_{2}^{\mathrm{i}, \mathrm{i}+1^{2}}+\tau_{2}^{\mathrm{i}-1, \mathrm{i}^{2}}\right) \\
\left.-\frac{2 \mathrm{e}^{\mathrm{i}}\left(1+v^{i}\right)}{15 \mathrm{E}^{\mathrm{i}}}\left(\tau_{1}^{\mathrm{i}, \mathrm{i}+1} \tau_{1}^{\mathrm{i}-1, \mathrm{i}}+\tau_{2}^{\mathrm{i}, \mathrm{i}+1} \tau_{2}^{\mathrm{i}-1, \mathrm{i}}\right)-\frac{2\left(1+v^{i}\right)}{5 \mathrm{E}^{\mathrm{i}}}\left(\mathrm{Q}_{1}^{\mathrm{i}}\left(\tau_{1}^{\mathrm{i}, \mathrm{i}+1}+\tau_{1}^{\mathrm{i}-1, \mathrm{i}}\right)+\mathrm{Q}_{1}^{\mathrm{i}}\left(\tau_{2}^{\mathrm{i}, \mathrm{i}+1}+\tau_{2}^{\mathrm{i}-1, \mathrm{i}}\right)\right)\right]
\end{array}\right.
\end{gathered}
$$$$
\mathrm{w}_{\mathrm{c}}^{\mathrm{i}}=\frac{1}{2}\left[\frac{1}{\mathrm{e}^{\mathrm{i}} \mathrm{E}^{\mathrm{i}}}\left(N_{11}^{i^{2}}+N_{22}^{i^{2}}+2 N_{12}^{i^{2}}\left(1+v^{i}\right)-2 v^{i} N_{11}^{i} N_{22}^{i}\right)\right.
$$$$
\left.+\frac{12}{e^{i^{3} E^{i}}}\left(M_{11}^{i^{2}}+M_{22}^{i^{2}}+2 M_{12}^{i^{2}}\left(1+v^{i}\right)-2 v^{i} M_{11}^{i} M_{22}^{i}\right)\right]
$$ 